\title{
Microscopic Characterization of Eco-friendly Lokta Paper
}

Girja Mani Aryal ${ }^{1}$, Washat Ware ${ }^{2}$, Shubo $\mathrm{Han}^{3}$, Gibin George ${ }^{2}$, Zhiping Luo ${ }^{3}$, Krishna Prasad Kandel ${ }^{1}$, Bhoj Gautam ${ }^{2}$ and Bhanu Neupane ${ }^{1}$

${ }^{1}$ Tribhuvan University, Kathmandu, Nepal, ${ }^{2}$ Fayetteville State University, Fayetteville, North Carolina, United States, ${ }^{3}$ Fayetteville State University, FAYETTEVILLE, North Carolina, United States

Lokta paper is a handmade paper indigenous to Nepal. The paper is made from inner fibrous bark of Lokta bushes; evergreen shrubs that grow in Himalayan forests ranging from 1600 to $4000 \mathrm{~m}$. Because of its durability and resistance to bugs and moulds, the paper in its original or modified from is being used in official documents, calligraphy, holy books, packaging materials, and even to make paper bills [1]. The paper is fabricated in local levels following the traditional eco-friendly method of fiber processing. Firstly, outer scaly bark from raw fiber is manually removed and cut to small pieces and soaked in cold water for 5-6 hours. The biomass is boiled in water for around 5-10 hours and then washed with ash or alkali solution. The softened fiber biomass is beaten to make pulp and then dispersed to make slurry. The slurry is poured in a wooden mesh frame or paper moulds over a water tank. The frame is then drained and air dried to get paper sheet. Depending on the demand, the paper can be colored using natural dyes obtained from different plants and or patterned for artistic purposes. A systematic characterization of lokta paper sheet from material perspective, which helps to explain it novel properties, is missing in literature. In this work, we studied fiber organization, morphology and elemental distribution in lokta paper sheet with the help of scanning electron microscope (SEM) coupled to an electron probe micro analyzer (EPMA). For SEM imaging the paper sample was sputter coated with carbon and imaged in a JEOL field-emission JXA-8530F EPMA equipped with an SDD X-ray energydispersive spectrometer (EDS). Finally, we also did atomic force microscopy (AFM) imaging in tapping mode to explore surface roughness and structural details in the sample at nanoscopic level. AFM was performed at ambient condition in tapping mode. Finally, the X-ray diffraction (XRD) data were collected at Braggs' angle $2 \theta$ ranging from 5 to $40^{\circ}$ by a X-ray diffractometer. The $\mathrm{Cu} \mathrm{K \alpha}$ line $\left(\lambda=1.540 \mathrm{~A}^{\circ}\right)$ was used as $\mathrm{X}$-ray source. The SEM micrographs of lokta paper imaged at different magnification is shown are figure $1 \mathrm{~A}-\mathrm{D}$. The micrographs show that in the paper the cellulosic fibers are randomly oriented so as to form densely packed interwoven networks (Figure 1 and B). It is known that strength of paper sheet is largely determined by strength of individual fiber and degree of cross linking within the individual fibers. The presence of long interconnecting fibers having no or low curl could provide durability and strength to the paper sheet. With further zooming individual fiber surface can be imaged (Figure 1C and D). We found fiber diameter in the range of 10-14 micrometer. In most of the fibers, individual micro-fibrils that run straight along the length of fiber are clearly visible (yellow dotted regions in Figure $C$ and D). In some fibers gummy material is found attached on the surface (white dotted region in Figure 1C. The micro-fibrils are visible due to removal of gummy materials such as lignin and hemicellulose from the fiber surface during processing. We found microfibril diameter and spacing in the range of 100-350 nm and 100-500 nm, respectively. The almost parallel arrangement of micro-fibril could provide excellent strength to individual fiber and to the paper sheet. The observation of micro-fibrils is also reported in fiber processed form other plant types and biomass [2, 3]. In a cellulose fiber amorphous and crystalline phases are known to exist in different proportions but these phases are not visible in SEM images. However, we observed the contribution from two cellulose forms in the XRD data (Figure 1E). The peak at $2 \Theta$ values of $\sim 16^{\circ}$ and $22^{\circ}$ originate from the crystalline planes (101) and (002), respectively. A broad background underlying the crystalline peaks originates form the amorphous regions [4]. 
We also did the EPMA analysis in the micro-regions labeled + in Figure $1 \mathrm{~A}$. We found $\mathrm{C}$ and $\mathrm{O}$ as the major elements along with other minor elements $\mathrm{Ca}, \mathrm{Fe}$, and $\mathrm{Cl}$ heterogeneously distributed in the paper. The elements $\mathrm{C}$ and $\mathrm{O}$ come from the cellulosic biomass, Ca could come from unwashed alkali or impurities in water, and $\mathrm{Fe}$ and $\mathrm{Cl}$ could come from water impurities. Figure 2 shows the AFM phase and topographic images of lokta paper. It is interesting to see the crystalline or quasi-crystalline regions on the surface (white boxes in figure 2A). These regions could be lignin and or other foreign materials precipitated on the fiber surface. The distinct stripes having cross section of $\sim 200-300 \mathrm{~nm}$ indicate the micro-fibril on the fiber. We see the roughness at the level of $\sim \pm 100 \mathrm{~nm}$ (figure $2 \mathrm{~B}$ ) which roughly corresponds to the height form top of a micro-fibril to the cleft adjacent to it. The measurement of force-distance curve that can provide the information on the elastic modulus in the different regions on individual fiber (fiber strength) could be subject of further study [5].

*Correspondence: bbneupane@cctu.edu.np, bgautam@uncfsu.edu

Acknowledgements: This work was supported by NSF PREM program DMR 1827731, EIR (ECCS-1900837), and DOD W911NF-09-1-0011.

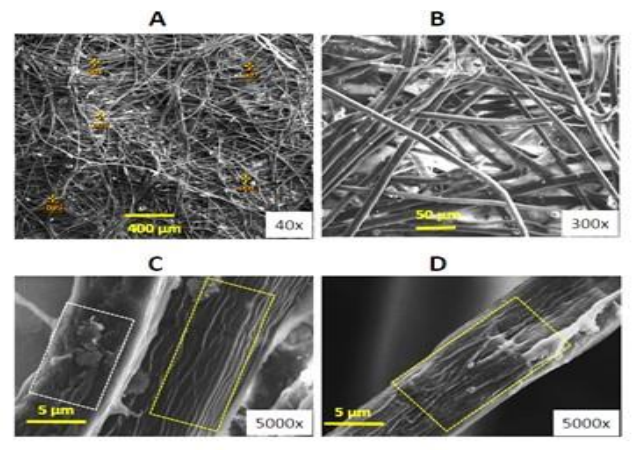

Figure 1. (A) SEM micrograph of the Lokta paper sheet measured at magnification of 40x, (B) 300x, (C) and (D) 5000x. Scale bar in A, B, C and D is 400, 50, 5, $5 \mu \mathrm{m}$, respectively.

A

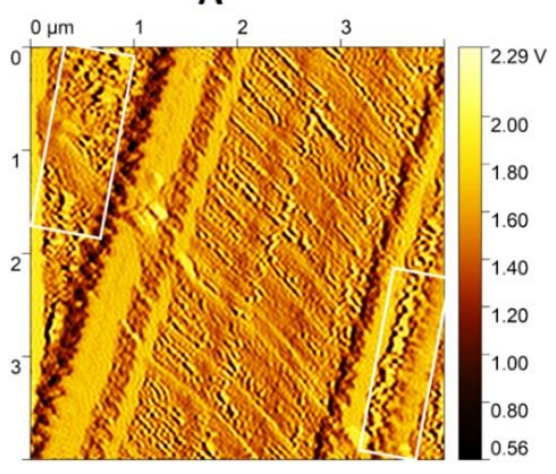

B

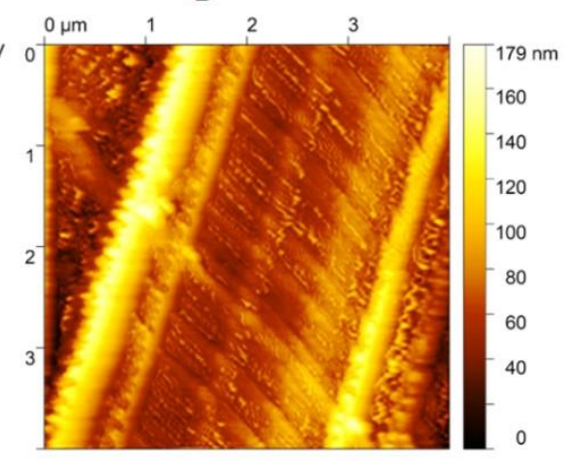

Figure 2. (A) Phase and (B) topographic AFM images of a small regions with a cellulose fiber.

\section{References}

[1] G. B. Banjara, German Technical Cooperation (GTZ), Handmade Paper in Nepal (2007).

[2] H. Sosiati et. al., International Conference on Physics 2014 (ICP 2014), p. 12

[3] H. Zhao et. al., Carbohydrate Polymers, 68 (2007), p. 235.

[4] S. Park, et. al, Biotechnology for Biofuels, 10 (2010), p. 1-10.

[5] R. R. Lahiji et. al., Lagmuir, 26(2010), p. 4480. 